\title{
Integration of Maintenance in the Tactical Production Planning Process under Feasibility Constraint
}

\author{
Martin Géhan, Bruno Castanier, and David Lemoine \\ Ecole des Mines de Nantes, \\ 4 Rue Alfred Kastler, 44300 Nantes, France \\ \{martin.gehan, bruno.castanier, david.lemoine\} @mines-nantes. fr
}

\begin{abstract}
This paper deals with the problem of the joint optimization of the master production schedule and maintenance strategy for a manufacturing system. An efficient production planning and maintenance policy will allow to minimize the impacts of the potential random failures and will let the plan to be feasible. We present a modelisation where we take into account a feasibility constraint; the optimization problem is formulated as a linear program. We propose a heuristic algorithm to solve it and we show the impact of the feasibility constraint on different criteria.
\end{abstract}

Keywords: production planning, maintenances, feasibility.

\section{Introduction}

To be sustainable, a manufacturing company should take the right decisions to satisfy on time the customer demands, while minimizing the production costs. Thus, the production planning should be determined: the production goals are fixed according to the estimation of the available resources for a midterm horizon. That involves to determine the lot-sizes to produce: the aim is to calculate in function of the estimated production capacity the quantities which should be produced for each item and for each period of the given horizon to satisfy the customer requests, at the lowest cost (including the production, holding and setup costs). Many mathematical models can be used to solve this problem, [5] gives a classification of these ones.

However, some authors [3] shown that the production capacity of the system is often wrongly estimated: there is a difference between the estimated capacity and the real available capacity during the production process. This gap can lead to the infeasibility of the production plans. The authors identified several factors for this problem, and underline the main one: the consumption of capacity by maintenance actions. They consider two kinds of capacity loses: the capacity consumed by the preventive maintenance actions (the system should be maintained regularly) and the one consumed by the corrective maintenance actions, which are carried out every time that a breakdown occurs during the production. The figure 1 illustrates how the failures which occur during production (so the corrective maintenances) turn the production plan to be infeasible. In order to minimize and to anticipate the impact of 


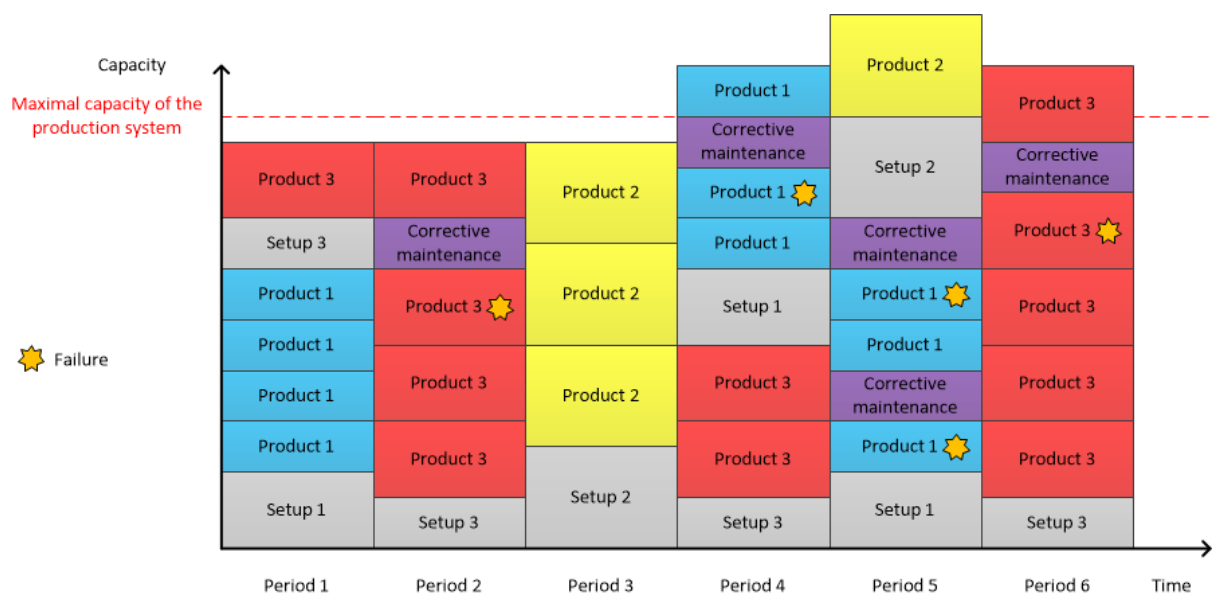

Fig. 1. Failures during production turn the production plan to be infeasible

the failures and of the maintenance actions on the production plan, making a joint planning of the production and the maintenance planning seems to be a reliable solution.

\section{Review of the Literature}

Tactical planning problems are usually classified according to several criteria: the number of items (single or multi items), the nature of the capacity (limited or unlimited), or the typology of the demand (constant or variable) [5]. This last criterion is known to be the most structuring one. Indeed we can sort the literature models into two categories:

- the Economic Production Quantities (EPQ) based models (where the demand is constant). The main objective is to find the fixed quantity to produce periodically which minimizes the total cost (including the storage costs and the setup costs) to meet the customers' demand. Generally, the production is resumed periodically when the concerned stock is empty,

- the Lot-Sizing Problem (LSP) based models (where the demand is variable, but known). Here, the temporal horizon is split in several periods, and the demand varies at each period. The aim is to find the quantities to be produced at each period in order to meet the customer demands while minimizing the total logistic cost.

We propose to focus on the study of the integration of maintenance concerns in the production planning when the demand is assume to be variable.

We found only nine papers devoted to the integration of maintenance into LSPbased models. [7] presents a model with an infinite production capacity with only one item, and a constant failure rate which implies no preventive maintenance. He studied 
two failures modes for which the price of the resuming of the production varies, and proposed a dynamic programming model to study the two cases and to provide a dynamic production plan. All the others authors $[1,8,10,12,4,9,6,2]$ propose to determine jointly a production plan and a preventive maintenance policy.

$[1,8,10]$ propose a policy of periodic maintenance where preventive maintenances can only be carried out at the beginning of the periods. This strategy is basic and takes into account only a single criterion: the time. The main advantage of the use of this method is its simplicity when applied: preventive maintenance dates are determined in advance (when the production plan is established), and not dynamically. However, the fact that this policy is periodic can lead to several problems: the lack of flexibility of the planning of the preventive maintenance does not allow to carry-out them at the optimal times, either maintenance are planned too often, that will generates a significant maintenance cost ; or not often enough, that will let the system degrades and will generate many failures during production. [12] propose a periodic maintenance policy, but this one is based one the produced quantities. [9] uses a similar policy: the preventive maintenances are planned into time windows, and these ones are periodical in the time. Even if this policy has the same drawbacks than the previous presented ones, it is more flexible and allow to profit a little bit more of the opportunities of the production plan for maintenance. For [6, 2] maintenance could be planned only at the beginning of the periods, but not necessary periodically. Finally [4] propose a model, where the failure rate depends of the degradation of the system, which increases randomly with the amount of produced quantities. They propose a method to determine jointly a degradation threshold for which a preventive maintenance is applied, and a production planning which the feasibility probability is parametrizable.

The proposed maintenance policies are based only on a single criterion, and most authors do not consider the problem of feasibility of production plans they establish. Developping new models taking into account a feasibility constraint, with a maintenance policy based on several criteria seems to be a interesting solution to answer to our problem.

\section{Problem Statement and Mathematical Formulation}

We consider a time horizon of $T$ periods. The production system can produce $N$ items, noted $i$. A the end of each period $t$, a quantity $d_{i, t}$ of each item $i$ should be available to satisfy the customer demand, shortages are not allowed. The production system, which is composed of only one machine, has a production capacity of $c_{t}$ at each period $t$. The production of an item $i$ consume $p_{i}$ units of capacity. The various setup operations which are needed to launch the production of the item $i$ consume $\tau_{i}$ units of capacity.

The system is ageing when it produced, the consequence of this ageing is the increase of the failure probability during the production. The age of the system increases by $p_{i}$ units when one item $i$ is produced. We note $A_{t}$ the age of the system 
at the end of the period $t$. The failures are assumed to be Weibull distributed, with the parameters $(\beta, \eta)$. The cumulative failure rate is noted $\Lambda$. We consider it increases with the system age, so $\beta>1$. During the production, the system is ageing from $a_{1}$ to $a_{2}$ (the capacity consumed by the production is therefore $a_{2}-a_{1}$ ), the cumulative hazard rate function is:

$$
\Lambda\left(a_{1}, a_{2}\right)=\int_{a_{1}}^{a_{2}} \frac{\beta}{\eta}\left(\frac{u}{\eta}\right)^{\beta-1} d u=\left(\frac{a_{2}}{\eta}\right)^{\beta-1}-\left(\frac{a_{1}}{\eta}\right)^{\beta-1}
$$

When a failure occurs, the production stops. A corrective maintenance should be carry out to resume the production. These corrective maintenances are classified as minimal: they do not affect the system age, which remain the same than after the failure. We assume that a corrective maintenance consumed $c_{c m}$ units of the capacity. When a period starts, a preventive maintenance can be carry out, these ones are considered perfect: they restore the age of the system to the new condition, so the failure rate becomes zero. The capacity consumed by these maintenances is $c_{p m}$.

At the end of each period, the manufactured products which are not dedicated to answer the demand of the current period can be stocked. In this case a unitary holding $\operatorname{cost} h_{i}$ should be payed for each unit of the item $i$. The stocked quantity of item $i$ at the end of the period $t$ is noted $I_{i, t}$. The setup operations involve a cost $s_{i}$ each time that the production of items $i$ is launched. A preventive maintenance involved a cost of pm, and we consider that corrective maintenances are costless. The aim of the problem is to minimize the sum of the production and maintenance cost.

We consider a feasibility constraint for the production plan. A period will be said to be $\varepsilon$-feasible if and only if the probability that the capacity consumed by all the production actions and by the maintenance actions (preventive et corrective) will be less than or equal to the available capacity of the period, is greater than or equal to a threshold $\varepsilon$.A production plan will be said to be $\varepsilon$-feasible if and only if each of its period is $\varepsilon$-feasible.

\section{Objective}

The decision problem is the joint determination of a production plan $\varepsilon$-feasible (therefore the determination of the quantities $Q_{i, t}$ to produce and the quantity $I_{i, t}$ to stock for each product for each period) and a preventive maintenance policy $\Pi$ (ie to determine when preventive maintenance should be carry out). The production plan and the maintenance policy should minimize the total cost of the production, which is the sum of the holding costs, the setup costs, and the preventive maintenances costs:

$$
z=\min \sum_{t=1}^{T}\left\{\sum_{i=1}^{N}\left(s_{i} X_{i, t}+h_{i} I_{i, t}\right)+p m M_{t}\right\}
$$




\section{Main constraints}

Our model consists the classic constraints of the CLSP [11]:

- the material balance equation (the stock of a period is equal to the previous period one, plus the produced quantities and minus the demand),

- $\quad$ the requirement of the setup operation to produce one item,

- the capacity constraint is included in the $\varepsilon$-feasibility constraint that we present further.

The following constraints ensure that $A_{t}$ is equal to the age of the system at the end of the period $t$ :

$$
\begin{gathered}
A_{t} \leq A_{t-1}+\sum_{i=1}^{N} p_{i} Q_{i, t} \quad \forall t \in[1, T] \\
A_{t} \leq \sum_{i=1}^{N} p_{i} Q_{i, t}+\left(1-M_{t}\right) \cdot M \quad \forall t \in[1, T]
\end{gathered}
$$

\section{Modelization and linearization of the $\varepsilon$-feasibility constraint}

As the failures follow a Poisson distribution with parameter $\Lambda$ and if we set $a=A_{t}-$ $\sum_{i=1}^{N} p_{i} Q_{i, t}$ the probability that $k$ failures occur when the system is ageing from $a$ to $A_{t}$ during the production is:

$$
P\left[N_{f}\left(a, A_{t}\right)=k\right]=e^{-\Lambda\left(a, A_{t}\right)} \cdot \frac{\Lambda\left(a, A_{t}\right)^{\mathrm{k}}}{k !}
$$

For a period $t$, the maximum number of failures that could occur is:

$$
N_{f_{\text {max }}}(t)=\left\lfloor\frac{c_{t}-\sum_{i=1}^{N}\left(p_{i} Q_{i, t}+\tau_{i} X_{i, t}\right)-c_{p m} M_{t}}{c_{c m}}\right\rfloor
$$

The $\varepsilon$-feasibility constraint for a period can be expressed as follow:

$$
P\left[\sum_{i=1}^{N}\left(p_{i} Q_{i, t}+\tau_{i} X_{i, t}\right)+c_{p m} M_{t}+c_{c m} N_{f}\left(A_{t}-\sum_{i=1}^{N} p_{i} Q_{i, t}, A_{t}\right) \leq c_{t}\right] \geq \varepsilon
$$

We can rewrite this probability as a function of the maximum number of failures allowed for the whole production (in terms of capacity):

$$
P\left[N_{f}\left(A_{t}-\sum_{i=1}^{N} p_{i} Q_{i, t}, A_{t}\right) \leq N_{f_{\max }}(t)\right] \geq \varepsilon
$$


And finally we have:

$$
\sum_{k=1}^{N_{f \max }(t)} e^{-\Lambda\left(A_{t}-\sum_{i=1}^{N} p_{i} Q_{i, t}, A_{t}\right)} \cdot \frac{\Lambda\left(A_{t}-\sum_{i=1}^{N} p_{i} Q_{i, t}, A_{t}\right)^{\mathrm{k}}}{k !} \geq \varepsilon
$$

\section{Resolution of the Model and Sensitivity Analysis}

We linearised the previous constraint and tried to solve our linear program with CPLEX. We could not solve any data sets: the memory consumed by CPLEX was too huge. Therefore we chose to develop an heuristic algorithm to solve it. In this section we will describe the heuristic resolution method we developed.

\section{Creation of an heuristic algorithm for the resolution}

As we do not allow shortage product, for some cases the problem can have no solution, or maybe a few number. We developed an heuristic algorithm which try to found those solutions. It carries out those three tasks until that a $\varepsilon$-feasible production plan will be found or that no production plan can be determined in the first task:

- calculate a production plan (not $\varepsilon$-feasible but including preventive maintenances), to do that we choose a simple but efficient preventive maintenance policy: the age of the system must not exceed the value $a_{\max }$; the solver calculates jointly the production planning and the maintenance one whom minimize the costs,

- determine if the production plan is $\varepsilon$-feasible and calculate the violation of the constraint (in terms of capacity for each period) if it is not,

- $\quad$ change the capacity of periods of a plan in order to try to make it $\varepsilon$-feasible

This algorithm is carried out for several values of $a_{\max }$ : we divided the set $\left[1, \sum_{t=1}^{N} C_{t}\right]$ (which is the set of the possible values for $a_{\max }$ ) into several partitions (the number of partitions is a parameter of the algorithm). The bounds of the partitions are the values that we tried for $a_{\max }$. When no $\varepsilon$-feasible production plan can be associated to a value, we divided the partition where the best solution was found and we launch again the algorithm for the new values. In this way we tried to find the values of $a_{\max }$ from which we deduce low cost production plans.

\section{Sensitivity analysis}

We present in this section the experiments we done with the developed method. As we cannot find any dataset in previous researches we decided to build our own ones. We propose to study the influence of the parameter $\varepsilon$ on different critera:

- the total cost,

- the mean number of preventive maintenances,

- the feasibility of the problem. 
The cost of the production increases with the value of the parameter $\varepsilon$ (because of the production levelling which makes the plan to become feasible). The sharp increase in the cost function presented in the figure 2 that occurs from the value $\varepsilon=0.8$ is interesting: the cost increases linearly until this point, then to obtain a better feasibility level for the production plan will be very expensive. The number of preventive maintenance also increases with the parameter $\varepsilon$ : it is necessary to regularly perform preventive maintenance to reduce the probability that failures occur and so increase the feasibility probability. The number of solutions decreases fast with the increase of the parameter $\varepsilon$, then the problem becomes impossible to solve because the $\varepsilon$-feasibility constraint becomes to strong.

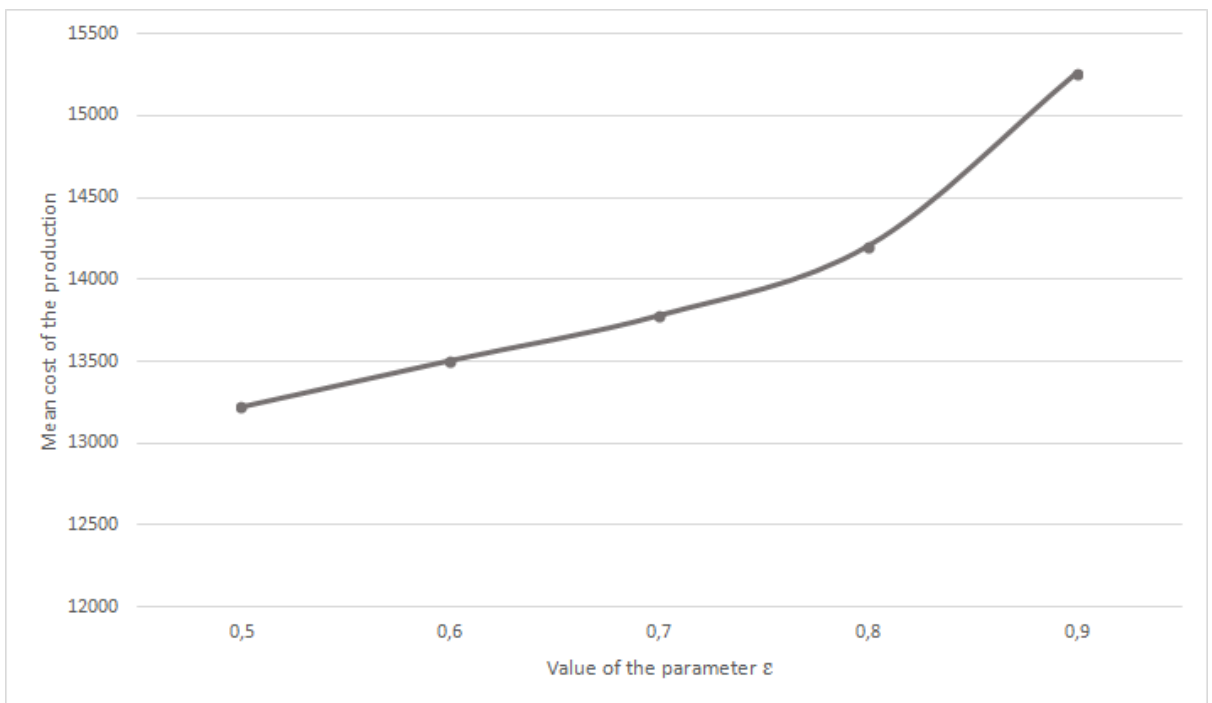

Fig. 2. Variation of the total cost of the production with the parameter $\varepsilon$

\section{Conclusion and Perspectives}

In this paper, we discussed about the problem of the integration of maintenance in the tactical planning process under a feasibility constraint. We presented a linear mathematical model, and explained why a classic solver could not solve it. Then we suggested an heuristic algorithm, this one used an age based policy which consist to apply a preventive maintenance before that the system reach a maximum age. Besides the interest of ensuring a feasibility level for the production plan, the joint optimization algorithm ensures a certain optimal aspect in terms of cost by the a priori allocation of capacity for the best suited maintenance according to the expected production levels. We test this algorithm on different benchmarks in order to evaluate 
the influence of the feasability parameter on different criteria, and shown that the insurance to have a good feasibility threshold for the production plan generate a significant cost.

\section{References}

1. Aghezzaf, E.H., Jamali, M.A., Ait-Kadi, D.: An integrated production and preventive maintenance planning model. European Journal of Operational Research 181, 679-685 (2007)

2. Aghezzaf, E.H., Najid, N.M.: Integrated production planning and preventive maintenance in deteriorating production systems. Information Sciences 178, 3382-3392 (2008)

3. Baglin, G., Bruel, O., Garreau, A., Greif, M., Kerbache, L., van Delft, C.: Management industriel et logistique. Economica (2005)

4. Castanier, B., Lemoine, D.: A preliminary integrated model for optimizing tactical production planning and condition-based maintenance. In: International Conference on Industrial Engineering and Systems Management (2011)

5. Comelli, M., Gourgand, M., Lemoine, D.: A review of tactical planning models. Journal of Systems Science and Systems Engineering 18, 204-229 (2008)

6. Fitouhi, M.-C., Nourelfath, M.: Integrating noncyclical preventive maintenance scheduling and production planning for a single machine. International Journal of Production Economics 136, 344-351 (2012)

7. Kuhn, H.: A dynamic lot sizing model with exponential machine breakdowns. European Joumal of Operational Research 100, 514-536 (1997)

8. Machani, M., Nourelfath, M.: A genetic algorithm for integrated production and preventive maintenance planning in multi-state systems. In: MOSIM 2010 (2010)

9. Najid, N.M., Alaoui Selsouli, M.M., Mohamed, A.: An integrated production and maintenance planning model with time windows and shortage cost. International Journal of Production Research 48, 2265-2283 (2011)

10. Nourelfath, M., Fitouhi, M.-C., Machani, M.: A genetic algorithm for integrated production and preventive maintenance planning in multi-state systems. In: MOSIM 2010 (2010)

11. Trigeiro, W., Thomas, L., Mc Clain, J.: Capacited lot sizing with setup times. Management Science 35, 353-366 (1989)

12. Weinstein, L., Chung, C.: Integrating maintenance and production decisions in a hierarchical production planning environment. Computers \& Operations Research 26, 1059-1074 (1999) 